\title{
Reactive power control strategies for
}

\section{UNIFLEX-PM Converter}

\author{
S. Pipolo, S. Bifaretti, V. Bonaiuto \\ Dept. of Industrial Engineering \\ University of Rome Tor Vergata \\ Rome, Italy
}

Abstract- The paper presents various control strategies for an advanced multilevel-based power converter. A Fuzzy Logic Control, based on power balance constrains and Proportional Reactive Power control based on a more rigorous approach, have been proposed. Two different ways to assign reactive powers have been illustrated for both the control strategies and then compared in Matlab/Simulink environment accounting two active power sets. Finally, advantages and drawbacks of the proposed strategies have been highlighted.

\section{INTRODUCTION}

Due to the increasing use of renewable energy sources and more in general distributed resources, the electricity distribution scenario is gradually evolving into an intelligent network type, so-called smart grids. New challenges in the energy system include not only the balancing of volatile, largely distributed, smallvolume energy production and consumption, but also develop smart and integrated networks, which function as components of a holistic energy system. Such an evolution needs active networks with interactive functionalities to integrate multiple energy sources and services, and enable consumers to use and produce energy more efficiently. Additionally, this requires new energy systems, which will enable decisions and the management of available energy production and distribution on the local level. A viable option that may be applied to facilitate the use of smart grids systems is certainly the implementation of "active nodes" using advanced specific configurations of power electronics converters. The active network architecture employs an increased number of power input nodes that can enable the direct routing of electricity [1].

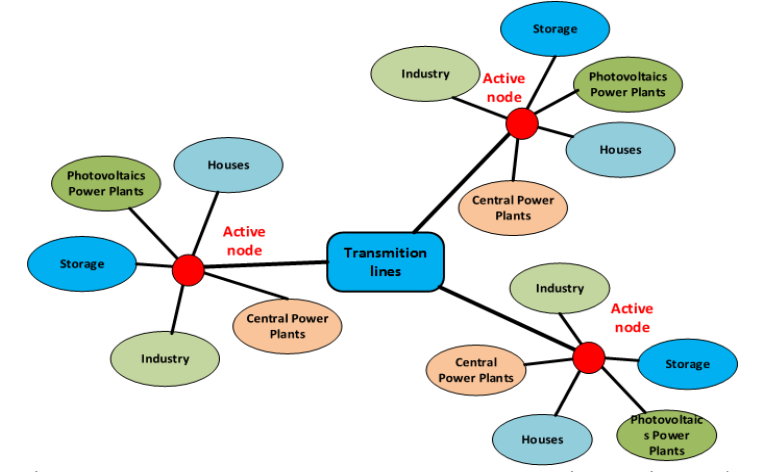

Fig 1. Smart energy management structure: overview active node.

Furthermore, the active node enables energy metering/monitoring as well as data processing from individual devices to permit the full operational control

\author{
L. Tarisciotti, P. Zanchetta \\ Dept. of Electrical and Electronic Engineering \\ University of Nottingham \\ Nottingham, UK
}

of the overall smart grid node by a Distributor System Operator (DSO). Fig. 1 schematically the concept of the smart energy network employing active nodes for the electricity routing. In this scenario, also due to the increasingly improved technology in terms of semiconductor devices and control techniques, different topologies of power converters have been developed suitable for this use in different contexts and at different power levels [2]-[3]. The main goal is to have a bidirectional power flow between the converter input/output sides as happens for $\mathrm{MV} / \mathrm{LV}$ solid-state transformers. If a DC-Link is available, it is possible to integrate easily the converter with storage systems thus becoming a multiport system.

In order to enable a more suitable use of the smart grid, it will be necessary in the near future to design advanced multiport converter topologies connecting simultaneously more users in both AC and DC. Only a few works focus on the possibility to connect more than two lines in AC. One of these has come out from the European project called "Universal and Flexible Power Management" (UNIFLEX-PM), which has shown a promising structure to manage the energy exchanges between three different electricity networks with a high power quality [4]. The main objective of the UNIFLEX-PM system is to provide a flexible and modular power electronic interface able to connect different kind of sources and loads, including MV electrical networks and energy storage system. Most of the studies about such a system concerned on the converter control techniques [5]-[6]. However, a fundamental aspect for a DSO is related to the realtime regulation of the active and reactive power flow. In [7] a control of Power flow profiles has been studied in different network conditions, but the UNIFLEX converter has been allowed to operate with only two ports. In [8] the limitations that the UNIFLEX configuration present when the converter works with all three ports in certain working conditions are investigated. In such conditions, the authors have shown that it is not possible to reach a unitary power factor, as it is necessary to supply a certain amount of reactive power to ensure the active power demand.

This paper has the aim to study an effective solution to distribute the reactive power amongst the three ports when these limitations occurs. In order to be able to enlarge as possible the operating powers range, two control strategies have been implemented and verified through accurate simulation models. 


\section{UNIFLEX CONVERTER}

The UNIFLEX converter structure is able to connect to a variety of loads and/or sources, including renewable energy sources or energy storage systems and interconnect multiple utilities in AC. The UNIFLEX 3port configuration concept is illustrated in Fig. 2. For example, one of the possibility is to connect Port 1 and Port 2 at medium voltage and Port 3 at low voltage.

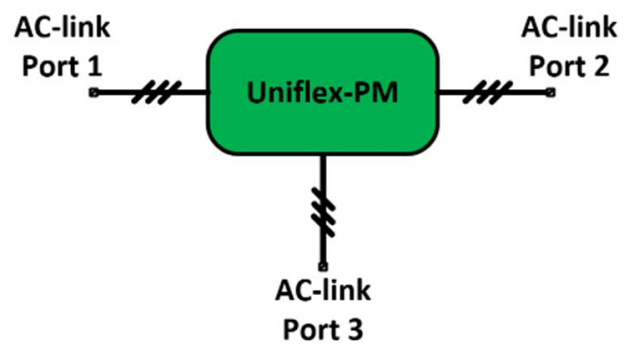

Figure 2. Block diagram of the UNIFLEX structure

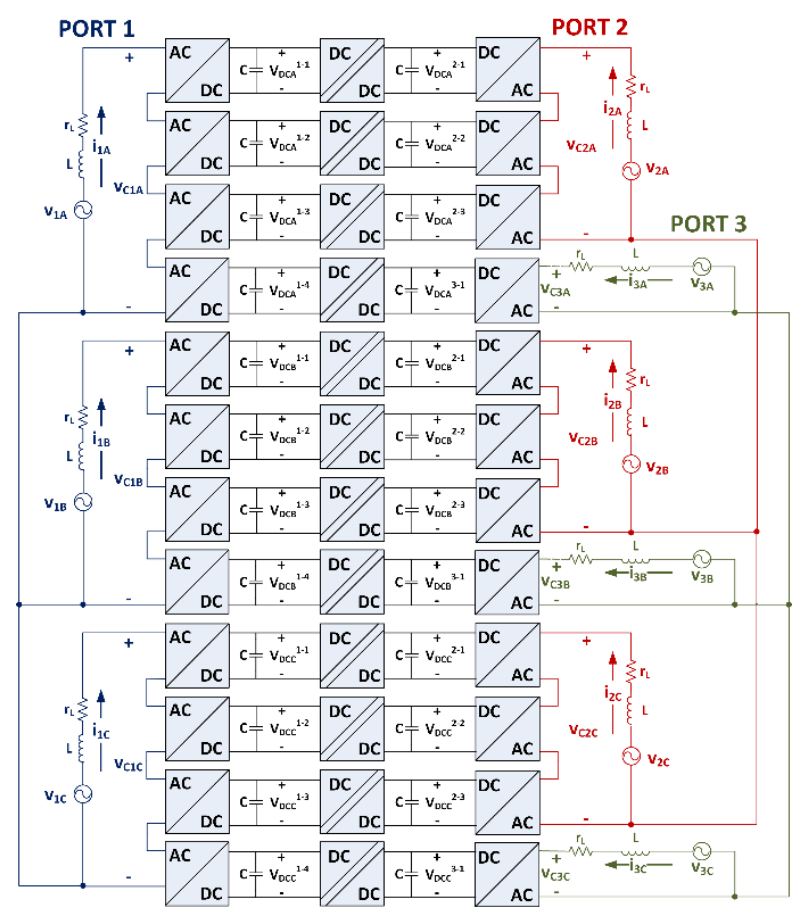

Figure 3. UNIFLEX detailed structure

The nominal parameters of the UNIFLEX-PM converter are shown in Table I.

\begin{tabular}{|c|c|c|c|}
\hline NAME & DESCRIPTION & VALUE & UNIT \\
\hline $\mathrm{C}$ & DC-Link capacitor & 3100 & {$[\mu \mathrm{F}]$} \\
\hline $\mathrm{R}_{\mathrm{L}}$ & Inductor resistance & 0.5 & {$[\Omega]$} \\
\hline $\mathrm{L}$ & AC filter inductance & 11 & {$[\mathrm{mH}]$} \\
\hline $\mathrm{P}^{\mathrm{NOM}}$ & Rated power & 5 & [MVA] \\
\hline $\mathrm{V}_{1}^{\mathrm{NOM}}$ & $\begin{array}{l}\text { Rated peak value of the } \mathrm{AC} \\
\text { supply on port } 1 \text { (line-to-line) }\end{array}$ & 3300 & {$[\mathrm{~V}]$} \\
\hline $\mathrm{V}_{2}^{\mathrm{NOM}}$ & $\begin{array}{l}\text { Rated peak value of the } \mathrm{AC} \\
\text { supply on port } 2 \text { (line-to-line) }\end{array}$ & 3300 & {$[\mathrm{~V}]$} \\
\hline $\mathrm{V}_{3}{ }^{\mathrm{NOM}}$ & $\begin{array}{l}\text { Rated peak value of the } \mathrm{AC} \\
\text { supply on port } 3 \text { (line-to-line) }\end{array}$ & 415 & {$[\mathrm{~V}]$} \\
\hline $\mathrm{V}_{\mathrm{DC}}^{\mathrm{NOM}}$ & Rated capacitor voltage & 1100 & [V] \\
\hline
\end{tabular}

\section{A. System limitation overview}

As mentioned above the UNIFLEX-PM converter is expected to control the power flow between the three ports at any power factor. However, an in-depth analysis have shown limitations on the power flow capability [8] which can be highlighted accounting the simplified model of one phase of Port 1 , shown in Figure 4.

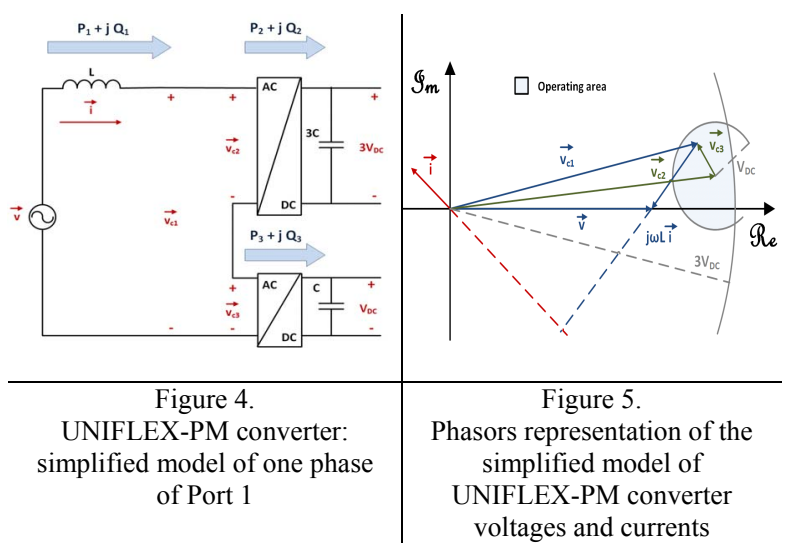

The AC/DC converter on Port 1 is divided in two converters: one 3-cell Cascaded H-Bridge enabling power flow to Port 2 and an H-Bridge connected to Port 3. As it can be noticed in Figure 6, the operating areas are divided into four zones according to the active power on port 2 and 3 . The red and green lines set the active power limits on Port 2 and on Port 3 beyond which a unity power factor operation is not allowed. In the operating areas $\mathrm{A}$ and $\mathrm{D}$ reactive power on Port 1 is not required, whereas, in the operating areas $\mathrm{B}$ and $\mathrm{C}$ it is necessary to provide a certain amount of reactive power [8]. The equations that link $P_{2}$ and $P_{3}$, identifying the operative areas, can be expressed as:

Bound A-B:

$$
P_{2-A B}=P_{3}\left(\frac{v_{d}}{V_{M A X}}-1\right)
$$

Bound C-D

$$
P_{2-C D}=P_{3}\left(\frac{3 V_{M A X}}{v_{d}-3 V_{M A X}}\right)
$$

where $V_{M A X}$ identifies the maximum output voltage of each H-Bridge and $v_{d}$ is the real part of the phasor While is always possible to control the active power flow on Port 1, the reactive power on Port 1, $Q_{l}$, can be arbitrarily chosen or imposed to provide enough circulating current to transport the power between the converter ports, depending on the operative point. $Q_{I}$ boosts the current amplitude so that the control is able to deliver powers demanded by Port 2 and Port 3 associated with the voltage $\bar{v}$ on Port 1 . 


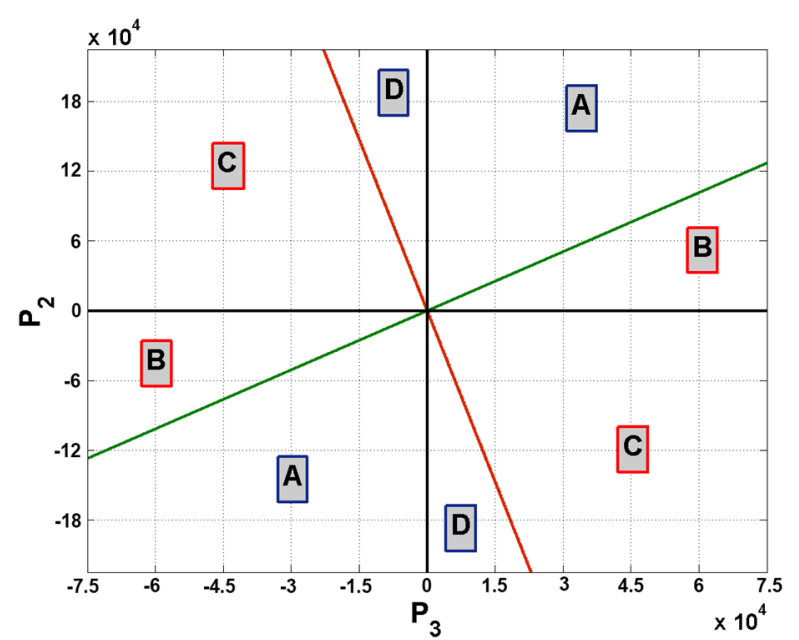

Figure 6. Port 1 operative conditions

However, in this way, the power factor on Port 1 is obviously degraded. The optimum reactive power required on Port 1 is located in shaded area in Figure. 5.

\section{REACTIVE POWER CONTROL STRATEGIES}

In the literature [8], several methods are proposed to appropriately share the reactive power:

- Proportional of active and apparent power

- Fixed share

The first option is to consider the apparent power $S$ proportional to the active power $P$ :

$$
\frac{S_{2}}{S_{3}}=\frac{P_{2}}{P_{3}}=k_{1}
$$

Replacing the apparent power as

$$
S_{i}=\sqrt{P_{i}^{2}+Q_{i}^{2}}
$$

where $i$ is the index refers to the generic port, the eq. (4) is obtained:

$$
\frac{Q_{2}}{Q_{3}}=\frac{P_{2}}{P_{3}}=k_{1} \rightarrow \quad Q_{2}=k_{1} Q_{3}
$$

Therefore, the reactive power is shared with the same proportion of the active power on the same port.

A second option is obtained, whereas the third port is constituted by a smaller number of H-Bridge compared with Port 2. In the present case Port 2 is constituted by three H-Bridge while Port 3 by a single H-Bridge.

As a result, it is possible to write:

$$
Q_{2}=k_{2} Q_{3}
$$

where $k_{2}$ is the ratio between the amount of H-Bridge used on Port 2 and those of Port 3.
Differently from eq.(4), in eq. (5) the ratio is independent of the value of the active powers. In [8] [9] it is suggested that having to fix at least two reactive power values, for example, to fix one of $Q_{I}$ and one of $Q_{2}$ and $Q_{3}$ obtaining the last reactive power from one of the proposed methods. However, considering the simplified circuit in Figure 4 it is possible to write the equation that links the reactive power as:

$$
Q_{1}=Q_{2}+Q_{3}+Q_{L}
$$

where $Q_{L}$ is a reactive power related to inductor $L$.

Substituting (4) into (6)

$$
Q_{1}=Q_{3}\left(k_{1}+1\right)+Q_{L}
$$

where

$$
Q_{3}=\frac{Q_{1}-Q_{L}}{\left(k_{1}+1\right)}
$$

The same can be done by replacing eq. (5) into eq. (6):

$$
Q_{3}=\frac{Q_{1}-Q_{L}}{\left(k_{2}+1\right)}
$$

Based on (8) and (9), it is evident that it is suitable to establish $Q_{1}$, to derive necessary $Q_{2}$ and $Q_{3}$ values for the proper operation of the converter.

\section{PROPOSED CONTROL}

Since it is sufficient to use only one of the reactive power value, it has been chosen to set $Q_{1}$ using two different approaches. The first approach is based on the Fuzzy Logic Control (FLC), whilst the second approach sets $Q_{l}$ based on the proportion between the required active power and the limits defined in Section II. In both cases, the $Q_{1}$ value is obtained, whereas $Q_{2}$ and $Q_{3}$ are set according to the considerations mentioned in the previous paragraph. In Figure 7, overall block diagram of proposed reactive power control is shown, where the logic control block identifies either of the two approaches mentioned above (FLC or proportional reactive power), and $Q 2$, Q3 calculator block is representative of eq. (3) and (7) for $k_{1}$ value and eq. (5) and (9) for $k_{2}$ value.

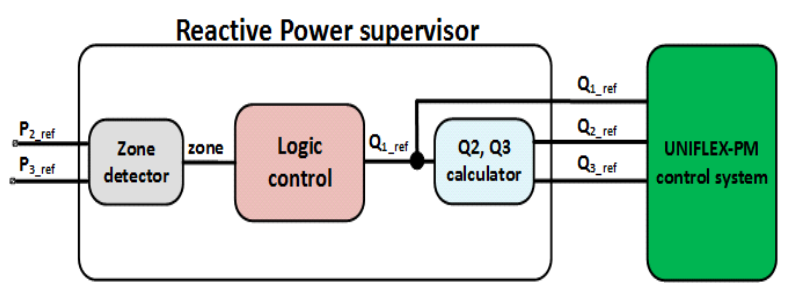

Figure 7. Block diagram of proposed reactive power control for UNIFLEX-PM converter 


\section{A. Method 1 - Fuzzy Logic Control}

The first method uses a fuzzy logic control, which aims to convert the control rules based on expert knowledge into a defined control strategy. It can be well suited to control systems with un-modeled dynamics. A Fuzzy Logic Control (FLC), based on the constrains deduced from Fig. 6, are implemented using the dedicated Matlab/Simulink tool. Two inputs have been defined for the Fuzzy Controller: active power on Port 2, $P_{2}$, and active power on Port $3, P_{3}$, to follow the working conditions represented in Fig. 6 . The output of the fuzzy controller is the reactive power on Port 1 . According to the rules written in the specific editor and shown in Table II, the controller takes the action and governs the amount of reactive power on Port 1 when the limitations (1) and (2) show in Section II occur. TABLE II. FUZZY RULES TO SET $Q_{1}$

\begin{tabular}{ccc|ccc}
$\boldsymbol{P}_{\boldsymbol{2}}$ & $\boldsymbol{P}_{\boldsymbol{3}}$ & $\boldsymbol{Q}_{\boldsymbol{1}}$ & $\boldsymbol{P}_{\boldsymbol{2}}$ & $\boldsymbol{P}_{\mathbf{3}}$ & $\boldsymbol{Q}_{\boldsymbol{1}}$ \\
\hline$P B$ & $P B$ & $\boldsymbol{Z}$ & $P S$ & $P B$ & $\boldsymbol{M}$ \\
$P B$ & $P S$ & $\boldsymbol{Z}$ & $N S$ & $N B$ & $\boldsymbol{M}$ \\
$N B$ & $N S$ & $\boldsymbol{Z}$ & $P S$ & $N S$ & $\boldsymbol{M}$ \\
$N B$ & $N B$ & $\boldsymbol{Z}$ & $N S$ & $P S$ & $\boldsymbol{M}$ \\
$P S$ & $P S$ & $\boldsymbol{L}$ & $P S$ & $N B$ & $\boldsymbol{H}$ \\
$N S$ & $N S$ & $\boldsymbol{L}$ & $N S$ & $P B$ & $\boldsymbol{H}$ \\
$P B$ & $N S$ & $\boldsymbol{L}$ & $P B$ & $N B$ & $\boldsymbol{H}$ \\
$N B$ & $P S$ & $\boldsymbol{L}$ & $N B$ & $P B$ & $\boldsymbol{H}$
\end{tabular}

In Table II letters $\mathrm{P}$ and $\mathrm{N}$ identify a positive and a negative values respectively; letters $\mathrm{B}, \mathrm{M}$ and $\mathrm{S}$ identify a big, medium and small values respectively; and finally letters $\mathrm{L}, \mathrm{H}$ and $\mathrm{Z}$ identify a low, a high or zero values. In $Q_{1}$ column, high value is set to $3 \cdot 10^{4}$ $V A r$, medium value is set to $2 \cdot 10^{5} \mathrm{VAr}$ and low value is set to $1 \cdot 10^{5} \mathrm{VAr}$.

\section{B. Method 2-Proportional Reactive Power}

The method sets the reactive power on Port 1 based on the limits set specified in equations (1) and (2). In particular, when the active power $P_{2}$ is less than the limit, the reactive power $Q_{1}$ is set according to the following relationship:

$$
Q_{1}=\left|P_{2}-P_{2 L}\right|
$$

where $P_{2 L}$ represents the limits specified in the equations (1) and (2). In fact $Q_{I}$ is proportional to the difference between $P_{2}$ and $P_{2 L}$, hence the method name Proportional Reactive Power [PRP].

\section{SIMULATION RESULTS}

Different tests have been performed in Matlab/Simulink environment accounting the two different power sets listed in Table III. The power set 1 is located in area B of Figure 6, while the power set 2 is located in zone $\mathrm{C}$. In zone $\mathrm{A}$ and zone $\mathrm{D}$, as mentioned in section II, it is not necessary to provide reactive power on Port 1; therefore, no power sets in these areas have been considered.
In Table III, $k_{1}$ and $k_{2}$ parameters are also indicated for the various sharing methodologies set out in section III. All simulations show the reactive power on all three ports in a range from $0.3 \mathrm{~s}$ to $0.7 \mathrm{~s}$. In particular, in the range between $0.3 \mathrm{~s}$ and $0.5 \mathrm{~s}$, the simulations are performed by setting $Q_{2}$ and $Q_{3}$ according to $k_{1}$ ratio, while in the interval $0.5 \mathrm{~s}-0.7 \mathrm{~s}$ simulations are performed according to $k_{2}$ ratio.

TABLE III. SET OF ACTIVE POWERS USED FOR THE SIMULATIONS

\begin{tabular}{||c|c|c|c|c|c||}
\hline \hline & $\begin{array}{c}\boldsymbol{P}_{1} \\
{[\boldsymbol{k W}]}\end{array}$ & $\begin{array}{c}\boldsymbol{P}_{2} \\
{[\boldsymbol{k W}]}\end{array}$ & $\begin{array}{c}\boldsymbol{P}_{3} \\
{[\boldsymbol{k W}]}\end{array}$ & $\begin{array}{c}\boldsymbol{k}_{1} \\
\mathbf{0 . 3 s}-\mathbf{0 . 5 s}\end{array}$ & $\begin{array}{c}\boldsymbol{k}_{2} \\
\boldsymbol{0 . 5 s}-\boldsymbol{0 . 7}_{\mathbf{s}}\end{array}$ \\
\hline $\begin{array}{c}\text { Set } \\
\boldsymbol{1}\end{array}$ & 115 & 45 & 70 & 0.6 & 3 \\
\hline $\begin{array}{c}\text { Set } \\
\mathbf{2}\end{array}$ & 85 & -135 & 50 & 2.7 & 3 \\
\hline
\end{tabular}

\section{A. Method 1 - Fuzzy Logic Control}

The following simulations have been performed with fuzzy logic control (FLC).

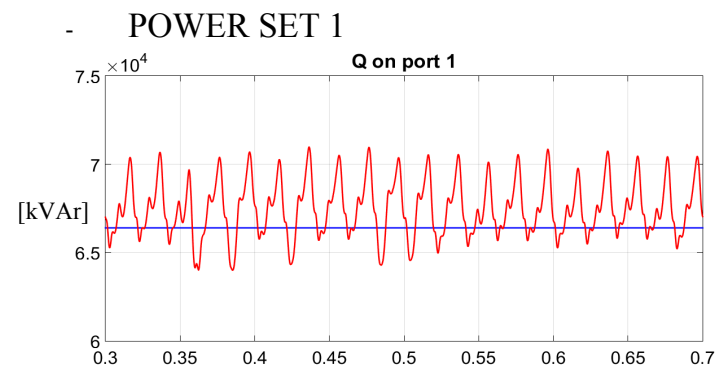

Figure 8. Reactive power $Q_{l}$ on Port 1 with FLC for power set 1

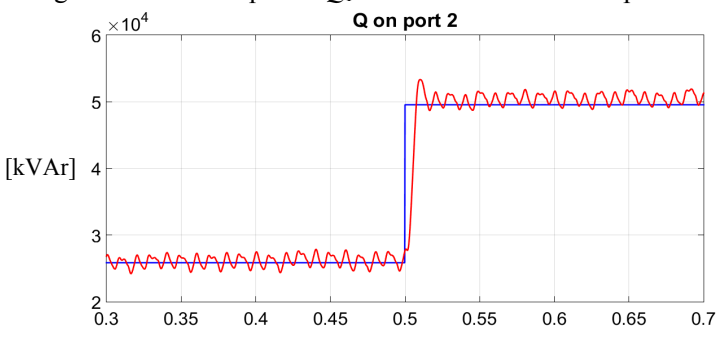

Figure 9. Reactive power $Q_{2}$ on Port 2 with FLC for power set 1

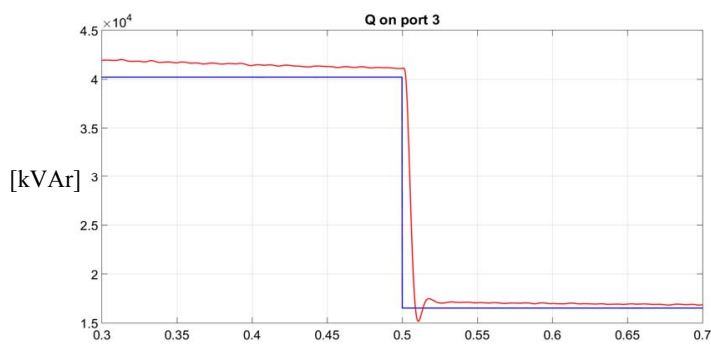

Figure 10. Reactive power $Q_{3}$ on Port 3 with FLC for power set 1 vdc Phase A

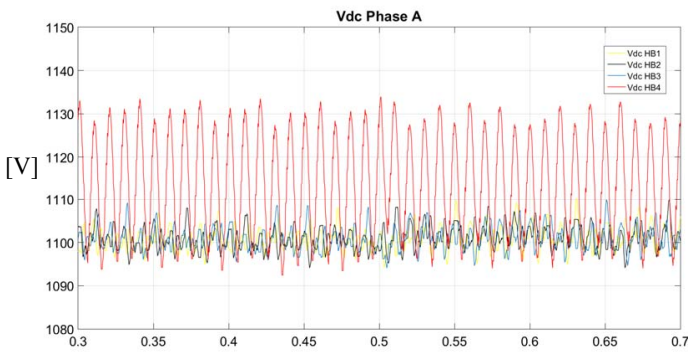

Figure 11. DC link voltage in phase A with FLC for power set 1 


\section{- $\quad$ POWER SET 2}

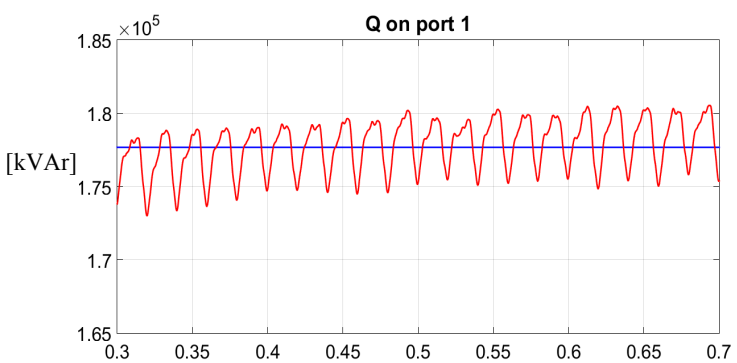

Figure 12. Reactive power $Q_{l}$ on Port 1 with FLC for power set 2

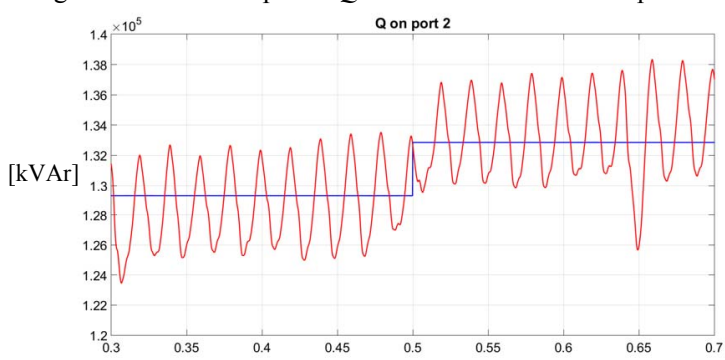

Figure 13. Reactive power $Q_{2}$ on Port 2 with FLC for power set 2

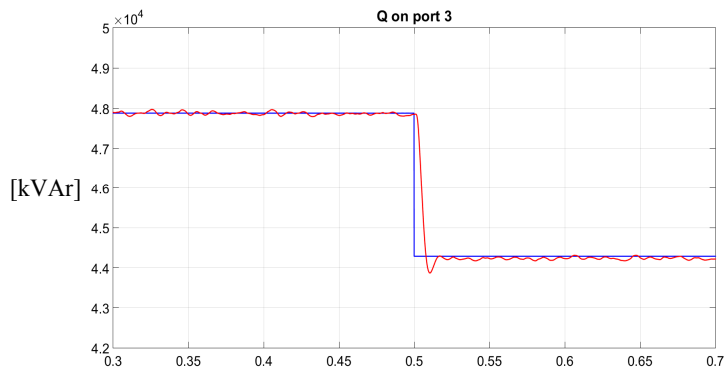

Figure 14. Reactive power $Q_{3}$ on Port 3 with FLC for power set 2

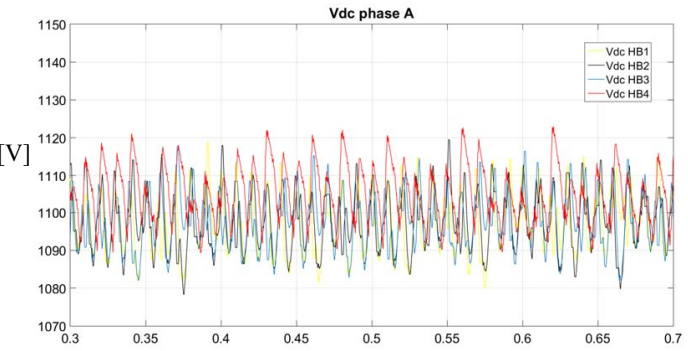

Figure 15. DC link voltage in phase A with FLC for power set 2

A. Method 2-Proportional Reactive Power [PRP]

\section{- $\quad$ POWER SET 1}

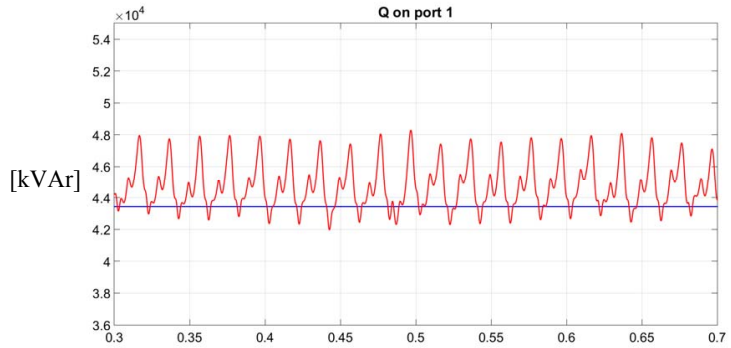

Figure 16. Reactive power $Q_{1}$ on Port 1 with PRP method for power set 1

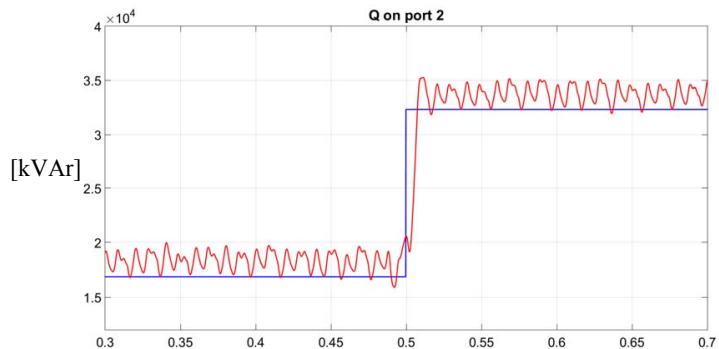

Figure 17. Reactive power ${ }^{0.45}$ on Port 1 with PRP method for power set 1

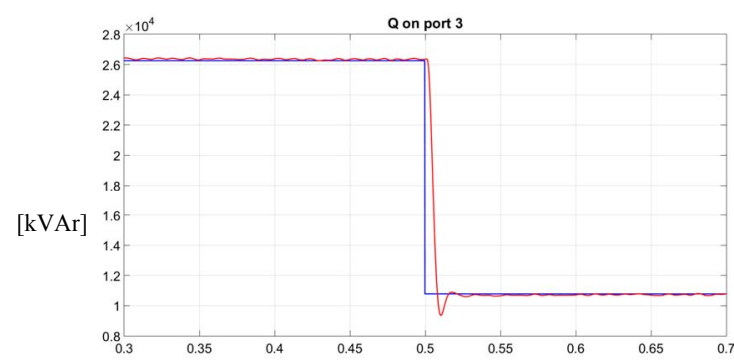

Figure 18. Reactive power $Q_{3}$ on Port 1 with PRP method for power set 1

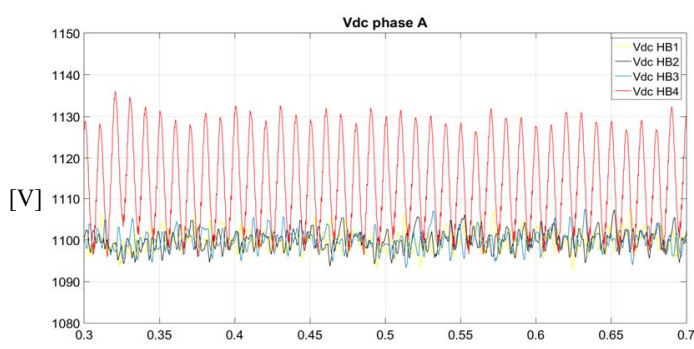

Figure 19. DC link voltage in phase A for power set 1

\section{- $\quad$ POWER SET 2}

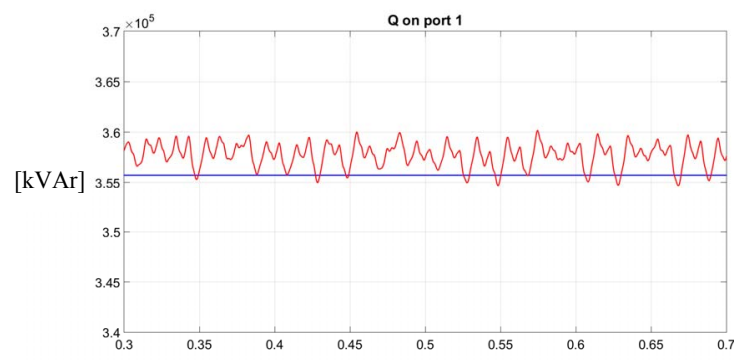

Figure 20. Reactive power $Q_{l}$ on Port 1 with PRP method for power set 2

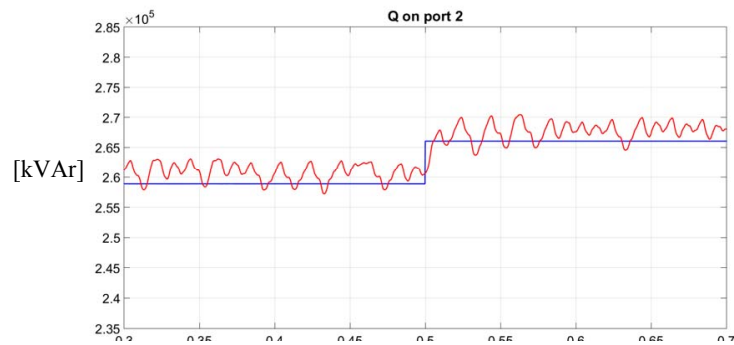

Figure 21. Reactive power $Q_{2}$ on Port 1 with PRP method for power set 2 


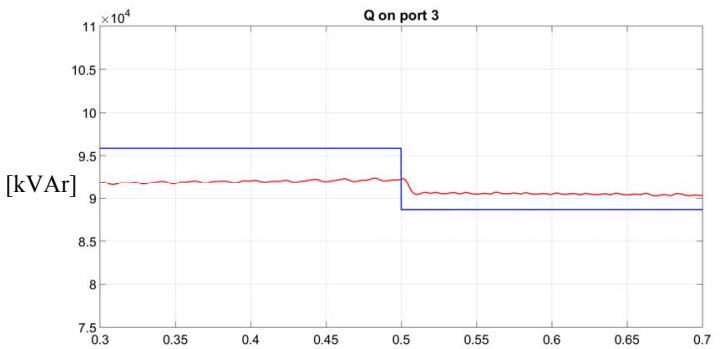

Figure 22. Reactive power $Q_{3}$ on Port 1 with PRP for power set 2

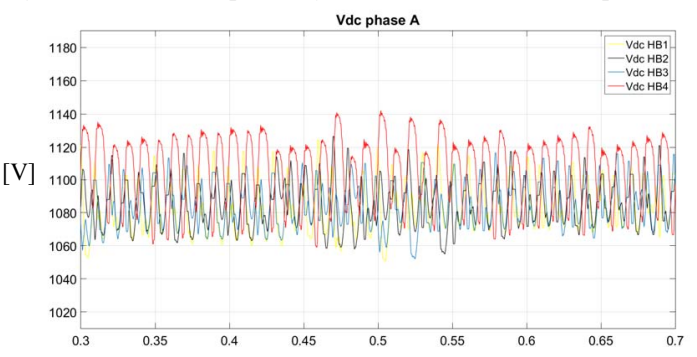

Figure 23. DC link voltage in phase A with PRP for power set 2

\section{DISCUSSION}

The value of $Q_{l}$ with the fuzzy control (Fig. 8) is about $65 \mathrm{kVAr}$, while it is of about $44 \mathrm{kVAr}$ with the PRP method (Fig. 16), consequently the power factor on the Port 1 is better in the latter case. The reactive power ripple is lesser than $4 \%$. Regarding the $k_{1}$ and $k_{2}$ parameters, it can be observed that with this powers sets, they are very different; $k_{1}=0.6$ and $k_{2}$ is fixed to 3 . Comparing Figure 11 and Figure 19 it can be observed that for the first set of powers and for both the proposed control methods and $k$ values, the ripple on the H-Bridge of the Port 3 is higher than other ports of about 15 Volts peak (less than 1.4\%). During the time interval between $0.5 \mathrm{~s}-0.7 \mathrm{~s}$, when the ratio between $Q_{2}$ and $Q_{3}$ is equal to $k_{2}$, the ripple is slightly lower, than the previous time interval relative to the constant $k_{l}$. The average value of $V_{d c}$ referred to the fourth $\mathrm{H}$ bridge (which feeds Port 3) is about $1115 \mathrm{~V}$ with respect to nominal $1100 \mathrm{~V}$, while the remaining $\mathrm{HB}$ remain to the average value of $1100 \mathrm{~V}$.

TABLE IV. POWER FACTOR OBTAINED FROM THE SIMULATIONS WITH FUZZY LOGIC CONTROL AND PROPORTIONAL REACTIVE POWER CONTROL

\begin{tabular}{|c|c|c|c|}
\hline \multicolumn{4}{|c|}{ FUZZY LOGIC CONTROL } \\
\hline & $\mathrm{PF}_{1}$ & $\mathrm{PF}_{2}$ & $\mathrm{PF}_{3}$ \\
\hline$K_{1} S E T 1$ & 0,87 & 0,87 & 0,87 \\
\hline \multirow[t]{2}{*}{$K_{1} S E T 2$} & 0,43 & 0,72 & 0,72 \\
\hline & & $\mathrm{PF}_{2}$ & $\mathrm{PF}_{3}$ \\
\hline$K_{2} S E T 1$ & 0,87 & 0,67 & 0,97 \\
\hline $\mathrm{K}_{2} \mathrm{SET} 2$ & 0,43 & 0,71 & 0,75 \\
\hline \multicolumn{4}{|c|}{ PROPORTIONAL REACTIVE POWER } \\
\hline & $\mathrm{PF}_{1}$ & $\mathrm{PF}_{2}$ & $\mathrm{PF}_{3}$ \\
\hline$K_{1} S E T 1$ & 0,94 & 0,94 & 0,94 \\
\hline \multirow[t]{2}{*}{$K_{1} S E T 2$} & 0,23 & 0,46 & 0,46 \\
\hline & & $\mathrm{PF}_{2}$ & $\mathrm{PF}_{3}$ \\
\hline$K_{2} S E T 1$ & 0,94 & 0,81 & 0,99 \\
\hline$K_{2} S E T 2$ & 0,23 & 0,45 & 0,49 \\
\hline
\end{tabular}

Analysing the second powers set, it is observed that the ripple on the $V_{d c}$, for all $\mathrm{H}$-Bridge, is greater in PRP control than the FLC control, regardless of the value of the constant of proportionality between $Q_{2}$ and $Q_{3}$, which in any case is very similar in both controls $\left(k_{1}=2.7 ; k_{2}=3\right) . \quad Q_{1}$ value, calculated with FLC, is halved compared with the same value calculated with PRP control, which leads to a clear improvement of the power factor in the case of FLC. By observing Figure 14 and Figure 22, it can be noticed that the reference of reactive power on the Port 3 is tracked more closely using the FLC control. In these conditions, it can certainly asserted that the FLC control shows better performance than the PRP control. Table IV illustrates the power factor obtained from the simulations presented in section $\mathrm{V}$.

\section{CONCLUSION}

Several reactive power control strategies have been presented for the UNIFLEX-PM 3-Port conversion system. A Fuzzy Logic Control, based on power balance constrains, and a Proportional Reactive Power control, that sets the value of reactive power on the port one basing on a more rigorous approach. Two different ways to assign reactive power on the Port 2 and on Port 3 have been illustrated for both the control strategies and then evaluated in Matlab/Simulink environment accounting two active power sets. The analysis has shown that, with all the proposed strategies, it is enough to fix only a value of reactive power instead of fixing two values of reactive power. It has been also highlighted that under certain working conditions, the FLC control provides better performance than the PRP control, while in other working conditions, the PRP control performs better. Thus, the development of a hybrid control strategy that integrates itself both the proposed methodologies will be the aim of a future work.

\section{REFERENCES}

[1] New ERA for Electricity in Europe. Distributed Generation: Key Issues, Challenges and Proposed Solutions European Commission, EUR 20901, 2003, ISBN 92-894-6262-0.

[2] Akagi, H. (2011). Classification, terminology, and application of the modular multilevel cascade converter (MMCC). Power Electronics, IEEE Transactions on, 26(11), 3119-3130.

[3] Babokany, A. S., Jabbari, M., Shahgholian, G., \& Mahdavian, M. (2012). A review of bidirectional dual active bridge converter. In 2012 9th International Conference on Electrical Engineering/Electronics, Computer, Telecommunications and Information Technology.

[4] F. Iov, F. Blaabjerg, J. Clare, P. Wheeler, A. Rufer and Andrew Hyde "UNIFLEX-PM - A Key-Enabling Technology for Future European Electricity Networks" EPE Journal, Vol 19 no 4, 2009.

[5] S. Bifaretti, P. Zanchetta, A. J. Watson, L. Tarisciotti, and J. C. Clare, "Advanced power electronic conversion and control system for universal and flexible power management," IEEE Trans. Smart Grid, vol. 2, no. 2, pp. 231243, Jun. 2011.

[6] Tarisciotti, L., Zanchetta, P., Watson, A., Bifaretti, S., \& Clare, J. C. (2014). Modulated model predictive control for a seven-level cascaded hbridge back-to-back converter. Industrial Electronics, IEEE Transactions on, 61(10), 5375-5383.

[7] Bifaretti, S., Zanchetta, P., Fan, Y., Iov, F., \& Clare, J. Power flow control through a multi-level H-bridge based power converter for Universal and Flexible Power Management in future electrical grids. In Proc. of 13th Power Electronics and Motion Control Conference, 2008. EPE-PEMC 2008, pp. 1771-1778.

[8] Dang, H. Q. S., et al. "Advanced integration of multilevel converters into power system." 2008 34th Annual Conference of IEEE Industrial Electronics. 2008.

[9] Report on converter structures - UNIFLEX-PM project deliverable D3.1, http://www.eee.nott.ac.uk/uniflex/Deliverables.htm 